\title{
INDEX AND LOCALISATION IN SIGN LANGUAGE: INTERFACE REQUIREMENT IN SEARCH OF FEATURES
}

\author{
Samar Sinha
}

This paper analyses INDEX and LOCALISATION as interface requirements owing to different $S$-M system that creates differences in terms of their features. It aims to provide an account of the structure building feature(s) associated with various instances of the phenomena in Indian Sign Language. The corpus is collected through fieldwork with the Deaf Associations, schools, and communities in India. The methodology employed is deductive following Branson \& Miller (1997) and Mathur (2000) (see Sinha 2008 for details). This paper argues that INDEX and LOCALISATION are associated with D head with $d$ features, and provides the ground from which cross-linguistic and cross-modality studies as well as investigations on universal set of features and their values can proceed.

Keywords: index, localisation, reference frame, feature, value

\section{Introduction}

The centrality of feature in syntax upholds the view that cross-linguistic as well as crossmodality similarities and differences in linguistic structures can be analysed in terms of features. This tenet seems to be promising in the study of various aspects of structure in sign language which have no parallel in spoken language. In sign language literature, such phenomena are generally ascribed to the 'modality differences' that the sign language employ.

This paper aims to address the 'modality differences' do not make sign language unique by virtue of its S-M system, rather the interface requirements of the S-M system creates the differences in terms of the feature, and INDEX refers to such feature(s) in Indian Sign Language (henceforth, ISL). The paper is divided into six broad sections. In section 2 , the facts relating to INDEX and LOCALISATION in ISL are described. A summary of the previous analyses of INDEX in sign language literature is provided to highlight the inadequacies in the explanation of the phenomenon in section 3. It is followed by newer observations from ISL. In the following section, the paper draws a feature based decomposition of INDEX and LOCALISATION in ISL. Section 6 concludes the paper.

\section{INDEX and LOCALISATION}

In the sign language literature, an INDEX refers to a type of pointing gesture that the signer uses to point to someone or something either manually and/or non-manually with the eye gaze or the body orientation; and the act as indexing. It is used to refer to an object, referents, spatial location, or events around the signer and the addressee in a common observable space, which I refer to as 'real reference frame.'

In ISL, a signer has an option to refer to a referent located in the real reference frame either by articulating the sign for the referent in its every instance or by indexing to the particular referent. In contrast, in the absence of an appropriate referent in the real reference frame, the signer articulates the sign for a referent and assigns it a distinct, specific locus in the signer's signing area through indexing. Such locus is termed as R(EFERENTIAL) LOCUS. I refer to such a reference frame as 'abstract reference frame' opposed to 'real reference frame,' and the united one as 'linguistic reference frame.'

In the sign language literature, the articulation of the sign and the assignment of a particular RLOCUS to it is called LOCALISATION (see Sinha 2008: 143). A sign can be localised by virtue of articulating the sign in the desired locus, without being followed by an INDEX. I shall recall these two types of LOCALISATION by descriptive labels manual LOCALISATION and non-manual LOCALISATION, respectively. In ISL, manual LOCALISATION involves the articulation of the HAND-SHAPE: G or B above with or without eye gaze. It can be effected on either the ipsilateral or

Nepalese Linguistics, vol. 34, 2019, pp. 73-80. 
74 / INDEX and LOCALISATION...

the contralateral side of the signer following a sign to be localised. ${ }^{1}$

(1)

$\begin{array}{lc}\text { eg:i } & \text { torso:i } \\ \text { M-A-D-A-N M-U-N-A IX-c } & \text { LIKE } \\ \text { "Madan likes Muna." } & \end{array}$

In (1), a signer localises MADAN non-manually with an eye gaze at the ipsilateral side of the signer. This is followed by manual LOCALISATION of MUNA on the contralateral side. In the articulation of like, the signer orients his/her body towards the ipsilateral side facing the R-LOCUS associated with MUNA. The subsequent INDEX to the R-LOCI at the ipsilateral and the contralateral sides suffices to refer to MADAN and MUNA, respectively.

In ISL, however, not all nouns allow LOCALISATION: only [+animate], [+concrete], [+locative] nouns may be localised, and [+abstract], [+mass], and [+generic] nouns may not. It shows that there is semantic restriction on LOCALISATION. Despite such restrictions, once a referent is localised resulting an R-LOCUS, an INDEX to the R-LOCUS suffices to refer to the same referent unambiguously in the discourse as shown in (2), even after many intervening signs like adjective that occur in between the noun and the LOCALISATION.

(2) ANCIENT-PAST KING BRAVE IX-i. IX-i FEM-BORN THREE HAVE. OLD.

"Once upon a time, there was a brave king. He had three daughters. (He was) old."

In non-manual LOCALISATION, the referent is either localised by the eye gaze, or by employing the strategy of role play in the discourse. In the latter, the narrator often plays the role of the characters in turn and narrates the event from the character's perspective as direct speech. This is either carried out by the signer orienting his/her body towards the character's R-LOCUS or by taking the perspective of the agent or experiencer of the event.

\footnotetext{
${ }^{1}$ Zeshan (2003) mentions that INDEX can either precede or follow a sign it localises. However, the earlier instance is not observed in my corpus.
}

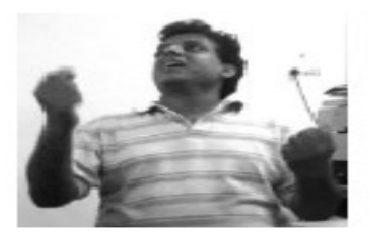

(a)

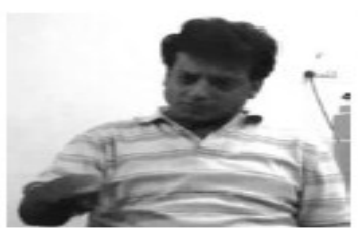

(b)
Figure 1: Role play

In Fig. 1(a), the signer plays the role of a driver; hence sitting. In Fig. 1(b), the signer's role is of a standing police officer, who stops the car. The role play shows various spatial dimensions that the referents actually occupy in the discursive context.

To sum up, the ISL data shows that LOCALISATION and INDEX are two different phenomena with different syntactic and semantic restrictions although similar in the articulatory terms.

\section{Previous analyses}

In sign language literature, the phenomena of LOCALISATION and INDEX are, often, regarded as one; hence, they are analysed as the same. As a consequence, LOCALISATION is subsumed under INDEX and has barely received any distinct linguistic analysis, whereas INDEX is most often ascribed as pronoun citing its anaphoric function as seen in (2). Most often an INDEX to the locus of the signer and the addressee are analysed as first and second person, respectively, and an INDEX to the actual referent and/or to the R-LOCI is analysed as third person. It is in this trifurcated linguistic reference frame, an INDEX is analysed as a pronoun.

There are, however, several other analyses of the phenomenon in various sign languages. Friedman (1975) argues that person reference in American Sign Language (ASL) is accomplished through the use of indexing, but maintains that there is no equivalent of pronoun in the ASL lexicon. LilloMartin and Klima (1990), although agrees that there is only one pronoun listed in the sign language lexicon, argues for a referential index, which is overtly realised in sign language due to a specific effect of the modality, in contrast with those of spoken language. Bahan (1996), MacLaughlin (1997) associate LOCALISATION, 
INDEX and possessive ASL sign with the head of the determiner phrase. Neidle et al. (2000: 31) claim that 'spatial locations constitute an overt instantiation of phi-features (specifically, person features) in ASL.' Pfau and Steinbach (2006; 2011; also see Pfau 2011) propose the grammaticalization of pointing gesture to locative, locative marker, demonstrative pronoun, personal pronoun, relative pronoun, agreement marker and agreement auxiliary.

On the other hand, there are authors who contest the claim that indexing has a pronominal function. Ahlgren (1990) argues that Swedish Sign Language INDEX areessentiallydemonstratives. McBurney (2002: 365) argues that in sign language "the coding of participant roles is accomplished not through abstract categories of person, but rather through gestural deixis." Schlenker (2011: 9), claims that "...it would be an overstatement to claim that all the features of sign language pronouns are analogous to those of their spoken language counterparts." ${ }^{2}$

In ISL, Zeshan (2003) has regarded INDEX and LOCALISATION as the same following its functions. She views that "the index is also used to localize a referent in sign space, that is, to indicate a point with which the referent is to be associated in the following text" (Zeshan 2003: 165). In other functions, when INDEX is used for deictic function and, anaphorically, it is considered to be equivalent to demonstratives and pronouns, respectively. Hidam $(2010 ; 2014)$ also favours person feature for ISL.

Despite several strains of analyses in the sign language literature, the syntactic and the semantic restrictions on INDEX and LOCALISATION are overlooked or obliterated. Consequently, the former is analysed as person, pronoun, demonstrative, etc., and the latter has barely received any attention in sign language literature. Such descriptive/analogical analyses often resort to the 'modality differences,' and fail to provide account of the phenomena.

\footnotetext{
${ }^{2}$ For various other approaches see Liddell (2000), Sandler and Lillo-Martin (2006), Schlenker et al. (2013), Schlenker (2014) and Kuhn (2016).
}

4. INDEX and LOCALISATION: a fresh observation from ISL

Sinha $(2003,2006,2008)$ acknowledges the fact that there are syntactic and semantic restrictions on INDEX and LOCALISATION, and makes a distinction between the two in ISL. Further, he observes a contrast in LOCALISATION of place names. The place name in which the signer is situated at the moment of signing is localised differently from the place name in which the signer is not.

\section{(3) R-A-M IX-i BOMBAY IX-d DELHI IX-u d-GO-u}

"Ram will go to Delhi from Bombay."

(4) IX-s DELHI IX-d J-O-B f-GET-s

"I got a job in Delhi."

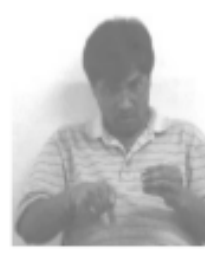

(a)

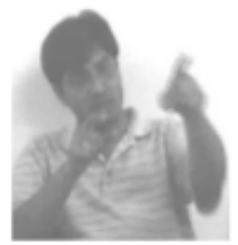

(b)

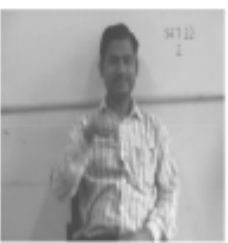

(c)
Figure 2: Localisation of place

In Fig. 2(a) and 2(b), the LOCALISATION of the sign BOMBAY and DELHI, respectively is shown from (3). In the former, the signer is in BOMBAY, so the sign is localised close to the signer; in the latter, LOCALISATION shows the spatio-temporal distance between the signer and the place localised i.e. DELHI. ${ }^{3}$ Contrast this with Fig. 2(c) from (4), the signer's spatio-temporal situation i.e. DELHI is localised closed to the signer unlike in (3). This shows that space is viewed from the perspective of the signer which creates an egocentric view (often referred to as origo), and the referents are in relation to the signer.

In addition to the heightened use of space, it is also found that the descriptive content alone is not enough to determine a unique referent and an INDEX must be articulated in ISL. INDEX is a

\footnotetext{
3 (3) and (4) were elicited at Mumbai (formerly,
} Bombay) and New Delhi, respectively. 
76 / INDEX and LOCALISATION...

demonstrative which can either precede or follow the noun sign as shown in (5).

\section{(5) a. BOOK IX GOOD \\ b. IX BOOK GOOD \\ "This/that book is good."}

In (5) INDEX has an overt deictic reading necessarily. However, it is not the case that each and every instance of an INDEX in ISL has necessarily deictic as well, apart from its referential reading. This contrasts with (6), shown below, where the deictic reading of IX i.e. BHUDEO) is inherent but implicit.

\section{IX-i FEM-BABUNI IX-c i-LIKE-c "(Bhudeo) likes Babuni."}

Further, Sinha (ibid.) discusses another phenomenon associated to INDEX and LOCALISATION which has barely received any attention in sign language literature. It is related to the dynamicity inherent to both reference frames, where referents may change discourse space-time. Consider the example from ISL in (7), concentrating on how the referent MONKEY is treated.
a. MONKEY IX-i BIG
"A monkey is big."
b. TREE-c i-CLIMB-c
"(The monkey) climbs a tree."
c. IX-c APPLE=EAT
"The monkey eats an apple (on the tree)."

In (7a), MONKEY is manually localised at the ipsilateral side of the signer (in the abstract reference frame). tree is articulated at the contralateral side of the signer showing an instance of non-manual LOCALISATION. In (7b), CLIMB has a path movement that has the onset $\mathrm{R}$ LOCUS associated with the MONKEY and the onset is at the top facet of the contralateral R-LOCUS associated with the TREE, respectively. The sign MONKEY is not signed nor INDEXED (as it is a discourse topic); the onset of the path movement suffices to refer to the monkey. The path movement shows that the MONKEY has climbed the TREE, and is at the top fact of the TREE. In (7c), MONKEY is not indexed where it is localised i.e. the ipsilateral R-LOCUS, but at the top facet of the TREE at the contralateral side of the signer.
The above example (7) indicates that even in the abstract reference frame as in the real reference frame, R-LOCI decay as events/situations unfold, and cease to be linguistically significant. This is exactly as would have been in the real reference frame for an identical sentence, except that instead of signing MONKEY in (7a), the signer would have merely pointed to the real-time referent MONKEY in the spatio-temporal coordinate. In other words, INDEX tracks the movement of the referent in the spatio-temporal dimension wherever the referent is at that point of discourse. This shows that the INDEX to the RLOCUS or the physically present referent in the partitioned signing space is not an invariant pronominal form as in spoken language. He further argues that had it been like a spoken language pronoun, the signer would have INDEX to the same R-LOCUS. In short, the dynamic referents inherent to both reference frames may change discourse space-time in ISL.

Sinha (ibid.), hence, argues that in ISL an INDEX to the R-LOCUs in the partitioned signing space is not a pronoun, but an R-expression, thereby involving reference of the Chandan type rather than the he type. He argues that every instance of INDEX in the linguistic reference frame is a noun (i.e. an R-expression), and that there does not exist a syntactic realisation of person other than the pragmatic/discourse roles. He, further, consolidates his analysis through the binding facts that support his claim.

\section{(8) MRIGE IX-i IX-i LIKE-i \\ "Mrige likes him." (lit. Mrige likes Mrige $_{i}$.)}

In the grammatical example (6), the R-LOCI associated with the ipsilateral localised referent refers to the same referent i.e. MRIGE. If the INDEX were indeed a pronominal, then Principle B of the Binding Theory is violated. The example is grammatical, however, suggesting that Principle B does not apply at all. Principle $\mathrm{C}$, that prohibits co-reference to hold between R-expressions, holds in a modified form in ISL by which c-commanded $\mathrm{R}$-expression are treated like epithets in spoken language as shown below in (9).

(9) John hired a secretary that hates the idiot. "John ${ }_{\mathrm{i}}$ hired a secretary that hates the idiot $\mathrm{i}_{\mathrm{i}}$. 
In ISL, there is no morphological realisation of any Case rather INDEX is used invariably. In genitive construction, compounding is employed as a strategy, where an INDEX followed by a possessum is grammatically valid genitive construction. It is, however, observed that HANDSHAPE: tA is often used for genitive/possessive opposed to INDEX, and to make distinction between the two forms.

To summarise the discussion so far, in both the real and the abstract reference frames, discourse relevant referents are treated alike as there is no fundamental syntactic distinction between the ${ }^{4}$ referents. In a discursive context, a signer can introduce a referent through LOCALISATION, and INDEX to the referent is equivalent to the articulation of the sign in its every instance. The communicative intent of INDEX would be of generic reference rather than a specific reference to an entity located in the real reference frame. The fact that INDEX to the R-LOCUS unambiguously refers to the same referent associated with the R-LOCUS even after many intervening signs shows that LOCALISATION does not signify an act of reference, whereas INDEX does, and the latter encodes deixis too.

\section{Decomposing D to $d$-features}

Acknowledging the fact that there is a distinction between INDEX and LOCALISATION, Sinha (2008) uses the ideas of Heim (1982) in distinguishing the two. On the basis of their semantics, Heim (ibid.) argues that indefinite descriptions are referential, and an indefinite is used to introduce a new entity into the discourse (Novelty Condition), while a definite or a pronoun is used to refer to old/familiar entities (Familiarity Condition). Sinha (2008) argues that LOCALISATION is governed by the Novelty Condition, but INDEX is in service of the Familiarity Condition. Thus, the former signals indefiniteness in ISL, while the latter yields definite descriptions in ISL. This entails that a noun introduced into a discourse without LOCALISATION is indefinite while the subsequent articulation of the same in the discourse is

\footnotetext{
${ }^{4}$ The handshape: tA is a loanword from English and ASL for fingerspelling 'S.'
}

definite. Syntactically, INDEX and LOCALISATION occupy the head of DP.

A large cross-linguistic study, however, shows that the determiner may not encode definiteness i.e. the same determiner can be used for novel referent as well as familiar referent. In Squamish, Malagasy, and Skwxwu7mesh among other languages (see Ghomeshi et al. 2009), all determiners can be used in novel or familiar contexts, regardless of whether they are deictic or non-deictic. Hence, the D-link features like familiarity and novelty (see Aboh 2003) do not suffice to determine the feature composition. The Skwxwu 7 mesh data provide us with evidence for three-way distinction: definite, indefinite and nondefinite. In Skwxwu7mesh, non-definites can be used in both novel and familiar cases, but behave much like definites in familiar contexts. Considering this cross linguistic variation, the definiteness can be valued as $[ \pm$ definite $]$ and [ \pm indefinite $]$.

Like value decomposition of definiteness, D(eterminer) head is not a monolithic element but composed of features (henceforth, $d$-features); and these features vary cross-linguistically as well as on cross-modality basis. The following phenomena in ISL $^{5}$ are examined to ascertain the $d$-features of INDEX and LOCALISATION.

In ISL, a solitary INDEX to a referent has semantics of singular, and two successive INDEX is to mark dual. It is known as transnumeral singular and transnumeral dual, respectively (descriptive labels are from Zeshan 2003). It is also observed that to mark two of the referents, the signer makes eye gaze to the referents and a suppletive HANDSHAPE: $\mathrm{V}$ is used to include the two. Such suppletive form is labelled as dual. Another articulatory strategy to include more than two referents is to articulate a semi/circular horizontal arc sweeping the referents. This articulatory strategy with ARC is an instance of plural INDEX. Another strategy to mark plurality is successive INDEX to referents to include as many those referents as INDEX refers to. It is labelled as

\footnotetext{
${ }^{5}$ For details of each phenomena see Sinha (2008, 2013).
} 
transnumeral plural. The difference between transnumeral plural and the ARC is that the former is specific and the latter is not. The similar applies to transnumeral dual and dual. A schematic diagram sums up the phenomenon.

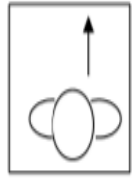

(a)

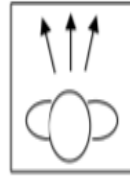

(b)

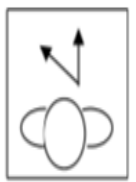

(c)

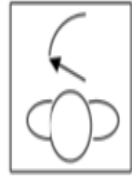

(d)

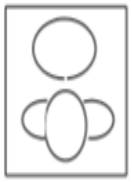

(e)
Figure 3: Number marking in ISL

Fig. 3(a) shows the INDEX to the addressee. Fig. 3(b) and 3(c) are an instance of transnumeral plural and dual, respectively. Fig. 3(d) has an ARC showing the inclusion of all the referents along with the sweep of the arc. In Fig. 3(e), all the referents in the discourse including the signer is included.

This establishes that number is among the $d$ features, and it is manifested with distinct values. Thus, the number feature in ISL is devised as [singular] and [augmented] following Harbour (2007) and Nevins (2006). Interestingly, ISL number interacts with specificity as discussed above. Hence, [ \pm specificity] is an additional feature along with number.

In ISL, gender differences are encoded for animate humans. The animate usually has FACE, if the signs (abbreviated in text as MASC. and FEM.) are not articulated. MASC. and FEM are articulated with the HANDSHAPE: $G$ at the LOCATION: PHILTRAL COLUMN and LOCATION: NOSE GROVE, respectively. They are obligatorily used for nondiscourse participants and may be localised. B are INDEX is also used for animate human masculine, and also for neuter.

Note that ISL display difference in terms of animacy; and Harley Ritter (2002) independently argued that animacy is a form of gender. Following Harley's (2008) two-feature system for distinguishing gender, ISL gender can be stated as $[ \pm$ feminine $]$ and $[ \pm$ neuter $]$, where $[+$ feminine, neuter] is fem; [-feminine, -neuter] is masc; and [feminine, +neuter] is neuter. In turn, this feature system captures the difference of animacy with $[ \pm$ neuter $]$.
It is also observed that HANDSHAPE: B in lieu of HANDSHAPE: $G$ is also used by the signers for INDEX as well as ARC. It is mostly used for a human referent since to point with HANDSHAPE: G is considered unmannerly in the many SouthAsian socio-cultural settings. HANDSHAPE: B, therefore, can be best understood as a marker of honorificity. Consequently, the manual INDEX HANDSHAPE: B and HANDSHAPE: G can be divided into honorific and non-honorific, respectively. It is also observed that honorific distinction is maintained in singular and dual masculine and feminine whereas the same distinction is not distinguished morphologically in dual.

Adger and Harbour (2008) appropriate honorificity within the ambit of phi-features. Based on ISL data, the honorific distinction can motivate to build feature distinction based on [ \pm honorific].

Zeshan ( $m s$.$) observes that ISL signs localized in$ the upper space involves entities that are invested with some degree of authority such as the government, the police and schools. Similar observation is expressed by Schlenker et al. (2012) for ASL and LSF, and maintains that this distinction of high or low position of a locus in signing space has a direct semantic reflex, akin to the semantic contribution of gender features of pronouns.

In spoken languages like in Burmese, Thai and Japanese, it is found that there are distinct forms in the pronominal system to indicate status, intimacy, and non-restraint, closely linked with other factors like politeness or respect, and also assertiveness which are directly connected with speech roles - the speech role of 'being an honoured addressee' as distinct from that of 'being an addressee of the same or lower status' (Bhatt 2004:112). In sign language, the high and the low loci which are associated with reference to the signer can be understood as the spatial representation of the speech roles as observed in the spoken languages. Accordingly, the high and the low loci can be introduced in terms of feature speech role with $[ \pm$ status $]$ values.

Recall examples (3-6) from the earlier section. The sign in Fig. 2(a) and 2(c) are used for location of the utterance and 2 (b) is used for location that 
is not the location of the utterance. This shows that ISL encodes a contrast in terms of visibility with [-visible] and [+visible] values. In addition to visibility, INDEX in ISL has the function of a demonstrative which can either precede or follow the noun sign as seen in (5) and (6). The deictic nature of demonstrative encode information about the location of the referent with respect to the utterance location that is visible. Since there are no defined loci in space to distinguish proximate, medial and distal but a continuum; for the ease of exposition, the relevant values associated with $[+$ visible $]$ are $[ \pm$ proximate $]$ and $[ \pm$ distal $]$.

On the basis of the above discussion and a wider cross-linguistic generalisations, the $d$-feature associated with INDEX and LOCALISATION in ISL is decomposed into following features and their values:
1) Number:
[ \pm singular $]$
[ \pm augmented]
2) Gender:
[ \pm feminine $]$
3) Honorificity: [ \pm honorific]
4) Visibility: [-visible] [ \pm proximate $]$
5) Speech role: [ \pm status $]$
6) Definiteness: $[ \pm$ indefinite $] \quad[ \pm$ definite $]$
7) Specificity: [ \pm specific $]$

Finally, each instance of INDEX and LOCALISATION is the realisation of those features along with their values.

\section{Conclusion}

This paper acknowledges the syntactic and the semantic restrictions on INDEX and LOCALISATION, and makes a distinction between the two in ISL. Rather than a 'modality difference,' it argues that INDEX and LOCALISATION signal indefiniteness and definiteness, respectively; and are associated with $\mathrm{D}$ head. Further, it decomposes the $\mathrm{D}$ head into $d$ features, and argues that each instance of INDEX and LOCALISATION is the realisation of those features along with their values. The inter-face requirements of the S-M system creates the differences in terms of the feature, and INDEX and LOCALISATION refer to such features. Although the paper presents an initial feature based analysis of the phenomena; hopefully, it laid the ground from which cross-linguistic and cross-modality studies as well as investigations on universal set of features and their values can proceed.

\section{Acknowledgements}

I wish to thank Prof. Ulrike Zeshan, Dr. Sibaji Panda, Gopal, Debdatta, Dharmesh and Monica for the ISL data. I thank Prof. Philippe Schlenker, and Prof. Ayesha Kidwai for her elaborate comments and suggestions on the earlier drafts of the paper. I am also grateful to the audience in The Faculty of Language: Design and Interfaces (2013), Indian Institute of Technology, Delhi. All errors are of course my own.

$\begin{array}{ll}\text { Notation Conventions } \\ \text { eg } & \text { Eye gaze } \\ \text { torso } & \text { Torso tilt } \\ - & \text { Fingerspelling } \\ \text { IX } & \text { INDEX } \\ \mathrm{c} & \text { Contralateral side of the signer } \\ \mathrm{i} & \text { Ipsilateral side of the signer } \\ \mathrm{u} & \text { Space above the signer's face } \\ \mathrm{d} & \text { Space below the signer's waist } \\ \mathrm{s} & \text { Signer } \\ \mathrm{f} & \text { Addressee } \\ = & \text { Incorporation } \\ d \text {-VERB- } u & \text { Path movement of verb }\end{array}$

References

Aboh, Enoch O. 2004. Topic and focus within D. Linguistics in the Netherlands, 21(1).1-12.

Adger, David and Harbour, Daniel. 2008. Phi Theory: Phi-Features across Modules and Interfaces. Oxford/New York: Oxford University Press.

Ahlgren, Inger. 1990. Deictic pronouns in Swedish and Swedish Sign Language. In Susan Fisher and Patricia Siple (ed.), Theoretical Issues in Sign Language Research 1, 167-174. Chicago: University of Chicago Press.

Bahan, B. 1996. Non Manual realisation of Agreement in American Sign Language. Boston: Boston University.

Bhatt, D.N.S. 2004. Pronouns. Oxford/New York: Oxford University Press.

Friedmann, Lynn A. 1975. Space, time and person reference in American Sign Language. Language, 51. 940-961.

Ghomeshi, Jila; Paul, Ileana and Wiltschko, Martina. 2008. Determiners: Universals and variation. Amsterdam/Philadelphia: John 
Benjamins Publishing Company.

Harbour, Daniel. 2007. Morphosemantic Number: From Kiowa Noun Classes to UG Number Features. Dordrecht: Springer.

Harley, Heidi and Ritter, Elizabeth. 2002. Person and number in pronouns: A feature-geometric analysis. Language, 78. 482-526.

Harley, Heidi. 2008. When is a Syncretism more than a Syncretism? Impoverishment, Metasyncretism, and Underspecification. In David Adger and Daniel Harbour (ed.), Phi Theory: Phi-Features across Modules and Interfaces, 251-294. Oxford/New York: Oxford University Press.

Heim, Irene. 1982. The Semantics of Definite and Indefinite NPs. Amherst: University of Massachusetts at Amherst. (Ph.D.Thesis.)

Hidam, Gourshyam. 2010. Incorporation in IPSL. New Delhi: University of Delhi. (M.Phil. Dissertation.)

Hidam, Gourshyam. 2014. The Syntax of Word Order in IndoPakistani Sign Language. New Delhi: University of Delhi. (Ph.D. Thesis.)

Kuhn, Jeremy. 2016. ASL Loci: Variables or Features? Journal of Semantics, 33(3). 449491.

Liddell, Scot K. 2000. Blended spaces and deixis in sign language discourse. In David McNeill (ed.), Language and Gesture, 331-357. Cambridge: Cambridge University Press.

Lillo-Martin, Diane and Klima, Edward. 1990. Pointing out differences: ASL pronouns in syntactic theory. In Susan Fisher and Patricia Siple (ed.), Theoretical Issues in Sign Language Research 1, 191-210. Chicago: University of Chicago Press.

MacLaughlin, Dawn. 1997. The Structure of Determiner Phrase: Evidence from American Sign Language. Boston: Boston University. (Ph.D. Thesis.)

McBurney, Susan. 2002. Pronominal reference in signed and spoken language: Are grammatical categories modality-dependent? In Richard P. Meier; Cormier, Kearsy and Quinto-Pozos, David (ed.), Modality and Structure in Signed and Spoken Languages. Cambridge: Cambridge University Press.

Neidle, Carol; Kegl, Judy and MacLaughlin, Dawn and Bahan, Benjamin and Lee, Robert G. 2000. The Syntax of American Sign
Language: Functional categories and hierarchical structure. Cambridge: MIT Press.

Nevins, Andrew. 2007. The representation of third person and its consequences for person-case effects. Natural Language and Linguistic Theory, 25(2). 273-313.

Pfau, Roland and Steinbach, Markus. 2011. Grammaticalization in sign languages. In Bernd Heine and Heiko Narrog (ed.), The Oxford Handbook of Grammaticalization. Oxford: Oxford University Press.

Pfau, Roland and Steinbach, Markus. 2006. Pluralization in sign and in speech: A crossmodal typological study. Linguistic Typology, 10. 135-182.

Pfau, Roland. 2011. A point well taken: On the typology and diachrony of pointing. In Gaurav Mathur and Donna J. Napoli (ed.), Deaf around the world, 144-163. Oxford: Oxford University Press.

Sandler, Wendy and Lillo-Martin, Diane. 2006. Sign Language and Linguistic Universals. Cambridge: Cambridge University Press.

Schlenker, Philippe; Lamberton, Jonathan and Santoro, Mirko. 2013. Iconic Variables. Lingbuzz/002564

Schlenker, Philippe. 2011. Donkey Anaphora: the view from Sign Language (ASL and LSF). Linguistics and Philosophy, 34(4). 341-395.

Schlenker, Philippe. 2014. Iconic Features. Natural Language Semantics, 22(4). 299-356.

Sinha, Samar. 2003. A Skeletal Grammar of Indian Sign Language. New Delhi: Jawaharlal Nehru University. (M. Phil. Dissertation.)

Sinha, Samar. 2006. Pronoun does not climb trees. Paper presented at the National Seminar on Language and Interfaces, University of Delhi.

Sinha, Samar. 2008. A Grammar of Indian Sign Language. New Delhi: Jawaharlal Nehru University. (Ph.D. Thesis.)

Sinha, Samar. 2013. Index in ISL: an interface requirement in search of features. Paper presented at The Faculty of Language: Design and Interfaces, Indian Institute of Technology, Delhi.

Zeshan, Ulrike. (ms.). Functions of the index in IPSL. Lancaster University.

Zeshan, Ulrike. 2003. Indo-Pakistani Sign Language Grammar: A typological outline. Sign Language Studies, 3(2). 157-212. 